\title{
Akkermansia muciniphila-derived extracellular vesicles influence gut permeability through the regulation of tight junctions
}

\author{
Chaithanya Chelakkot ${ }^{1,8}$, Youngwoo Choi ${ }^{2,8}$, Dae-Kyum Kim ${ }^{1}$, Hyun T Park ${ }^{2}$, Jaewang Ghim ${ }^{3}$, \\ Yonghoon Kwon ${ }^{2}$, Jinseong Jeon ${ }^{2}$, Min-Seon Kim ${ }^{4}$, Young-Koo Jee ${ }^{5}$, Yong S Gho ${ }^{2}$, Hae-Sim Park ${ }^{6}$, \\ Yoon-Keun $\mathrm{Kim}^{7}$ and Sung $\mathrm{H}$ Ryu ${ }^{1,2}$
}

The gut microbiota has an important role in the gut barrier, inflammation and metabolic functions. Studies have identified a close association between the intestinal barrier and metabolic diseases, including obesity and type 2 diabetes (T2D). Recently, Akkermansia muciniphila has been reported as a beneficial bacterium that reduces gut barrier disruption and insulin resistance. Here we evaluated the role of $A$. muciniphila-derived extracellular vesicles (AmEVs) in the regulation of gut permeability. We found that there are more AmEVs in the fecal samples of healthy controls compared with those of patients with T2D. In addition, AmEV administration enhanced tight junction function, reduced body weight gain and improved glucose tolerance in high-fat diet (HFD)-induced diabetic mice. To test the direct effect of AmEVs on human epithelial cells, cultured Caco-2 cells were treated with these vesicles. AmEVs decreased the gut permeability of lipopolysaccharide-treated Caco-2 cells, whereas Escherichia coli-derived EVs had no significant effect. Interestingly, the expression of occludin was increased by AmEV treatment. Overall, these results imply that AmEVs may act as a functional moiety for controlling gut permeability and that the regulation of intestinal barrier integrity can improve metabolic functions in HFD-fed mice.

Experimental \& Molecular Medicine (2018) 50, e450; doi:10.1038/emm.2017.282; published online 23 February 2018

\section{INTRODUCTION}

The digestive tract is home to trillions of microbes, termed the gut microbiota, which affect energy homeostasis, ${ }^{1}$ endotoxemia, ${ }^{2}$ metabolism and the immune response ${ }^{3}$ during various diseases; additionally, the gut microbiota establishes a symbiotic relationship with the host in healthy mice and humans. Recently, metagenomic studies have found that microbial communities differ between normal and obese subjects. ${ }^{4,5}$ The bacterium Akkermansia muciniphila may represent $3-5 \%$ of the microbial composition in the healthy human intestinal $\operatorname{tract}^{6,7}$ and have a crucial role in the regulation of the gut barrier and other homeostatic and metabolic functions. ${ }^{8}$ Studies have identified a loss in abundance of A. muciniphila in patients with obesity and type 2 diabetes (T2D). ${ }^{9,10}$ However, the precise physiological mechanisms affected by this bacterium during metabolic disorders and intestinal permeability regulation remain unclear.

The intestinal barrier is the major barrier that separates our body from the external environment and is essential for the maintenance of intestinal homeostasis and metabolism. ${ }^{11}$ Alterations in intestinal barrier integrity are closely associated with the onset of metabolic diseases. Obese individuals manifest metabolic endotoxemia as a result of increased intestinal permeability, and this is thought to be a crucial factor responsible for chronic inflammation. ${ }^{12,13}$ The gut microbiota has a critical role in contributing to intestinal

\footnotetext{
${ }^{1}$ Division of Integrative Biosciences and Biotechnology, Pohang University of Science and Technology, Pohang, Republic of Korea; ${ }^{2}$ Department of Life Sciences, Pohang University of Science and Technology (POSTECH), Pohang, Republic of Korea; ${ }^{3}$ NovaCell Technology Inc., Pohang, Republic of Korea; ${ }^{4}$ Asan Institute of Life Sciences, University of Ulsan College of Medicine, Seoul, Republic of Korea; ${ }^{5}$ Department of Internal Medicine, Dankook University College of Medicine, Cheonan, Republic of Korea; ${ }^{6}$ Department of Allergy and Clinical Immunology, Ajou University School of Medicine, Suwon-si, Republic of Korea and ${ }^{7}$ MD Healthcare Inc., Seoul, Republic of Korea

${ }^{8}$ These authors contributed equally to this work.

Correspondence: Dr Y-K Kim, MD Healthcare Inc., 9 World Cup buk-ro, 56-gil, Mapo-gu, Seoul 03923, Republic of Korea.

E-mail: ykkim@mdhc.kr

or Professor SH Ryu, Department of Life Sciences, Pohang University of Science and Technology, POSTECH Biotech Center, Pohang, Gyeongbuk 37673, Republic of Korea.

E-mail: sungho@postech.ac.kr

Received 4 July 2017; revised 9 September 2017; accepted 11 September 2017
} 
permeability and metabolic endotoxemia-induced inflammation in patients with obesity and diabetes. ${ }^{14}$

Extracellular vesicles (EVs) are lipid bilayer structures secreted from the gut microbiota, including from both Gram-negative and -positive bacteria. ${ }^{15,16}$ A variety of bacteria constitutively produce EVs, defined as spherical lipid bilayers with an average diameter of $20-200 \mathrm{~nm} .{ }^{17}$ EVs are composed of proteins, lipids, nucleic acids, lipopolysaccharides and other virulence factors associated with pathogenesis. ${ }^{18-20}$ Recent evidence suggests that EVs released by bacteria have diverse roles in the microbial community by transferring genetic material and proteins from the bacteria to the host. ${ }^{21} \mathrm{EVs}$ can also directly interact with immune cells and epithelial cells to initiate several signaling pathways and can be considered as functional units of the gut microbiota that mediate hostpathogen interactions. In addition, our previous study observed that EVs affected glucose metabolism by inducing insulin resistance. EVs affected the insulin signaling pathway, resulting in the inhibition of Akt phosphorylation. ${ }^{22}$ However, the unique mechanisms by which EVs regulate intestinal permeability and glucose homeostasis are not fully understood.

In this study, we evaluated the mechanism underlying the influence of A. muciniphila-derived EVs (AmEVs) on gut barrier function. AmEV treatment increased tight junction protein expression in Caco-2 cells and ultimately improved intestinal barrier integrity in high-fat diet (HFD)-induced diabetic mice in an AMPK-dependent manner. These results suggest that EVs are an important moiety in the regulation of gut permeability and metabolic functions by A. muciniphila.

\section{MATERIALS AND METHODS}

\section{Materials}

A. muciniphila bacteria (ATCC BA-835) were purchased from the American Type Culture Collection (Manassas, VA, USA). The AMPK inhibitor, compound C (171260), was purchased from Merck (Boston, MA, USA).

\section{Ethics statement}

This study was carried out in strict accordance with the recommendations in the Guide for the Care and Use of Laboratory Animals of the National Institute of Health (Bethesda, MD, USA). The experimental protocols were approved by the Institutional Animal Care and Use Committee at POSTECH, Pohang, Republic of Korea (permit number: POSTECH-2015-0018). All animal experiments were planned to minimize suffering.

\section{Bacterial culture and EV isolation}

A. muciniphila (ATCC BAA-835) was cultured under anaerobic conditions $\left(99 \% \mathrm{~N}_{2}\right.$ at $\left.37^{\circ} \mathrm{C}\right)$ until the optical density at $600 \mathrm{~nm}$ reached 1.5 as previously described. ${ }^{7}$ The isolation of EVs was performed as previously described. ${ }^{23}$ Briefly, bacterial cultures were pelleted at $10000 \mathrm{~g}$ for $20 \mathrm{~min}$, and the supernatant was filtered through a $0.45-\mu \mathrm{m}$ vacuum filter. The filtrate was enriched using QuixStand (GE Healthcare, Little Chalfont, UK) and subsequently filtered through a $0.22-\mu \mathrm{m}$ bottle-top filter (Sigma-Aldrich, St Louis, $\mathrm{MO}$, USA). The filtrate was pelleted by ultracentrifugation in a $45 \mathrm{Ti}$ rotor (Beckman Coulter, Fullerton, CA, USA) at $150000 \mathrm{~g}$ for $2 \mathrm{~h}$ at $4^{\circ} \mathrm{C}$. The final pellets were resuspended in phosphate-buffered saline (PBS) and stored at $-80^{\circ} \mathrm{C}$. The EVs were analyzed by transmission electron microscopy, dynamic light scattering and sodium dodecyl sulfate-polyacrylamide gel electrophoresis (SDS-PAGE) followed by gel staining with Coomassie Brilliant Blue R-250.

\section{Mammalian cell culture}

Caco-2 cells were cultured in Dulbecco's modified Eagles medium supplemented with $10 \%$ fetal bovine serum, $4 \mathrm{~mm}$ glutamine, $1 \%$ nonessential amino acids, $4.5 \mathrm{gl}^{-1}$ D-glucose, $110 \mathrm{mgl}^{-1}$ sodium pyruvate and $1 \%$ antibiotic cocktail. ${ }^{24,25}$ For cell signaling experiments, Caco-2 cells were treated with $5 \mu \mathrm{g} \mathrm{ml}^{-1}$ lipopolysaccharide (LPS) with or without $1 \mu \mathrm{g} \mathrm{ml}^{-1} \mathrm{EV}$, and the cells were lysed after $4 \mathrm{~h}$ for cell signaling experiments. For dose-dependency studies, Caco-2 cells were treated with $0.1,1$ and $10 \mu \mathrm{g} \mathrm{ml}^{-1} \mathrm{EVs}$, and the cells were lysed after $4 \mathrm{~h}$. For time-dependent studies, Caco- 2 cells were treated with $1 \mu \mathrm{g} \mathrm{ml}^{-1} \mathrm{EV}$, and the cells were lysed at $0,2,4$ and $8 \mathrm{~h}$. Cell lysates were used to examine the protein expression by western blotting.

\section{In vitro permeability assay}

In vitro permeability was determined by measuring the flow of fluorescein isothiocyanate (FITC)-dextran across the apicobasolateral layer in a Caco-2 monolayer cell culture as previously described. ${ }^{26}$ Caco-2 cells were cultured on collagen-coated transwell chambers as mentioned above for approximately 3 weeks, and the media was replaced every 2 days to generate monolayers. Monolayer formation was confirmed by measuring the trans-epithelial electrical resistance $e^{27,28}$ using a Millicell ERS-2 epithelial cell volt-ohm meter (Merck Millipore, MA, USA). Once the trans-epithelial resistance reached a plateau, the cells were considered to be differentiated and organized in a tight monolayer. Caco-2 monolayers were treated with LPS, LPS+Escherichia coli EV or LPS+AmEVs. Untreated cells were used as controls. After $4 \mathrm{~h}$, the trans-epithelial electrical resistance was measured again to analyze the LPS- or EV-induced changes in intestinal permeability. The apical chamber was then washed with Hank's Balanced Salt Solution, and $1 \mathrm{mg} \mathrm{ml}^{-1}$ FITC-dextran was added to the apical chamber (0-h time point). After $6 \mathrm{~h}, 100 \mu \mathrm{l}$ of media was collected from the basal chamber, and fluorescence intensity at an excitation of $490 \mathrm{~nm}$ and an emission of $530 \mathrm{~nm}$ was measured in each sample using a spectrofluorometer (Varian Cary Eclipse, CA, USA). The concentration of FITC-dextran in the sample was calculated using a standard curve of FITC-dextran.

\section{Animal studies}

Animal experiments were performed using C57BL/6 mice (6-8-weekold males) maintained under a $12 \mathrm{~h}$ light/dark cycle with free access to food and water. Mice were fed a $60 \%$ HFD for 12 weeks. After inducing a diabetic phenotype in the mice, EVs were orally administered for 2 weeks. Animal body weight was measured at the indicated time points. For the glucose tolerance test, mice were subjected to fasting for $16 \mathrm{~h}$, followed by intraperitoneal injection with glucose at a dose of $1 \mathrm{~g} \mathrm{~kg}^{-1}$ body weight (G7021, Sigma-Aldrich). Blood glucose concentrations were measured at the indicated time points using a glucometer (Accu-Check Performa; Roche, Basal, Switzerland), and blood was collected from the tail vein. Animals were then killed, and the colons were removed for morphological analysis. 


\section{In vivo permeability assay}

In vivo intestinal permeability was measured using the FITC-dextran assay as previously described. ${ }^{26,29-31}$ Mice were fed with the HFD for 12 weeks, followed by an oral gavage of $10 \mu \mathrm{g}$ per mice AmEVs for a period of 2 weeks every other day. The animals were starved for $6 \mathrm{~h}$ prior to the start of the experiments. FITC-dextran (Sigma Aldrich \#46944) was orally gavaged at a dosage of $400 \mathrm{mg} \mathrm{kg}^{-1}$. Blood samples were obtained by retro-orbital bleeding, and the fluorescence intensity in the serum was measured at an excitation wavelength of $490 \mathrm{~nm}$ and an emission wavelength of $530 \mathrm{~nm}$ using a spectrofluorometer (Cary Eclipse Fluorescence Spectrophotometer; Varian, Palo Alto, CA, USA). FITC-dextran diluted in PBS was used to plot a standard curve, and the serum concentration of FITC-dextran was calculated. The experiment was conducted a minimum of three times, with a sample size of at least six mice per group.

\section{Immunohistochemistry}

Paraformaldehyde-fixed, paraffin-embedded tissue sections were stained using a Vectastain Kit (\#PK-6161; Vector Laboratories, Inc., Burlingame, CA, USA). After the removal of paraffin and dehydration, the tissue sections were washed in PBS, and the endogenous peroxidase activity was quenched using $0.3 \% \mathrm{H}_{2} \mathrm{O}_{2}$. Sections were blocked with $5 \%$ horse serum diluted in PBST $(0.02 \%$ Tween 20 in PBS) and incubated with primary antibodies against occludin (ab31721, Abcam, Cambridge, UK), claudin-5 (ab15106, Abcam) or zonal occludens- 1 (ab59720, Abcam) overnight at $4{ }^{\circ} \mathrm{C}$. The sections were then washed in PBST and incubated with the biotinylated secondary antibody for $2 \mathrm{~h}$ at $25^{\circ} \mathrm{C}$. A fluorescently labeled secondary antibody (Invitrogen, Carlsbad, CA, USA) was used for fluorescence staining. Nuclei were stained with 4',6-diaminido-2-phenylindole and visualized under a Leica DM750 light microscope (Leica Microsystems, Wetzlar, Germany).

\section{Immunoblotting}

To observe the cell signaling pathway, HFD and EV-treated colon tissue samples and EV-treated, serum-fasted Caco- 2 cells were treated with lysis buffer (20 mM Tris- $\mathrm{pH} 8,150 \mathrm{~mm} \mathrm{NaCl}, 1 \mathrm{~mm}$ EGTA, $1 \mathrm{~mm} \mathrm{MgCl}_{2}, 1 \%$ deoxycholate, $10 \mathrm{~mm}$ glycerophosphate and $10 \mathrm{~mm}$ pyrophosphate) supplemented with a protease inhibitor cocktail. Ten micrograms of protein samples was then separated by gradient SDSPAGE (6-16\%) and transferred to a nitrocellulose membrane. The membrane was then incubated with the following antibodies: antiAMPK (Upstate Biotechnology, Lake Placid, NY, USA), rabbit antioccludin (ab31721, Abcam), anti-phospho-AMPK, anti-AMPK (Cell Signaling Technology, Danvers, MA, USA) and anti-actin (MP Biochemicals, Santa Ana, CA, USA).

\section{Ex vivo imaging}

EVs were incubated with $5 \mu \mathrm{M} \mathrm{Cy} 7$ mono NHS ester for $1 \mathrm{~h}$ at $37^{\circ} \mathrm{C}$. Cy7-labeled EVs were isolated using ultracentrifugation and then injected orally into C57BL/6 mice. After $6 \mathrm{~h}$, the mice were killed, and tissues were acquired from the liver, fat, muscle, spleen and large intestine. Cy7 fluorescence was detected by IVIS spectrum, and radiant efficiency was measured using the Living Image 3.1 software (Perkin Elmer, Waltham, MA, USA).

\section{Clinical studies}

Consecutive patients who visited the Department of Endocrinology and Metabolism at the Asan Diabetes Center were enrolled. This study was approved by the Asan Medical Center Institutional Review Board
(No. DKUH201401002-HE002). Feces were dissolved in PBS and centrifuged at $340 \mathrm{~g}$ for $10 \mathrm{~min}$. Supernatant fractions were pelleted twice at $10000 \mathrm{~g}$ for $30 \mathrm{~min}$ and filtered through a $0.22-\mu \mathrm{m}$ syringe filter (Sartorius Stedim Biotech, Goettingen, Germany). The filtrates were then subjected to density-gradient centrifugation in a Beckman ultracentrifuge (Beckman Coulter) at $100000 \mathrm{~g}$ for $2 \mathrm{~h}$ at $4{ }^{\circ} \mathrm{C}$. EVs were prepared by centrifugation at $200000 \mathrm{~g}$ for $2 \mathrm{~h}$ at $4{ }^{\circ} \mathrm{C}$ using a Beckman ultracentrifuge and stored at $-80^{\circ} \mathrm{C}$.

\section{Metagenomic analysis}

The $16 \mathrm{~S}$ rDNA analysis was performed as previously described. ${ }^{23}$ Briefly, 16S rDNA amplicons were subjected to pyrosequencing using the 454 GS FLX system (Roche, Basel, Switzerland). DNA sequences were identified using the ExTaxon database, after which a metagenomic analysis was performed by the ChunLab Corporation (ChunLab Inc., Seoul, South Korea).

\section{Statistical analysis}

Clinical EV data were normalized to the fecal data to eliminate the effects of individual microbiota. The empirical $P$-values were then calculated as described in a previous study. ${ }^{32}$ The heat map based on these data was drawn using MatLab 2015. The experimental data are expressed as the mean \pm s.e.m. Statistical significance was calculated using two-tailed Student's $t$-test or analysis of variance with Dunnet's multiple comparison correction, where two or more groups were compared. Differences with $P<0.05$ were considered statistically significant. Graphs were prepared using the GraphPad Prism V6.01 software (GraphPad Software, La Jolla, CA, USA).

\section{RESULTS}

AmEVs are relatively abundant in fecal samples of healthy humans compared with those of T2D patients

The gut microbial composition has a crucial role in the maintenance of host health, and a balance of interactions between gut microbes and host cells is essential for intestinal homeostasis. It is widely accepted that there is a drastic alteration in the gut microbial composition between healthy individuals and patients suffering from metabolic disorders, including obesity and T2D. EVs are functionally important mediators of host-pathogen interactions and are thus expected to differ between healthy and obese/diabetic individuals. Here we analyzed the fecal EV composition of healthy and diabetic subjects. Demographics and characteristics of the study subjects are shown in Supplementary Table S1. We isolated EVs from human feces (Supplementary Figure S1) and performed metagenomic analyses for both feces and the corresponding EVs from healthy controls and T2D patients. In feces from T2D patients, Firmicutes were dominant compared with those from healthy controls (Figure 1a). However, the composition of EVs was different from that of the feces samples, as the $16 \mathrm{~S}$ rDNA of Proteobacteria was frequently detected (Figure 1b). Further analysis of the bacterial population in EVs indicated that these EVs were composed of diverse species. Because each individual had different microbiota, the bacterial EV content was normalized by fecal bacterial content. When comparing the normalized bacterial EV content between healthy controls and T2D patients, EVs from A. muciniphila were detected only in healthy controls (Figure 1c). 
a

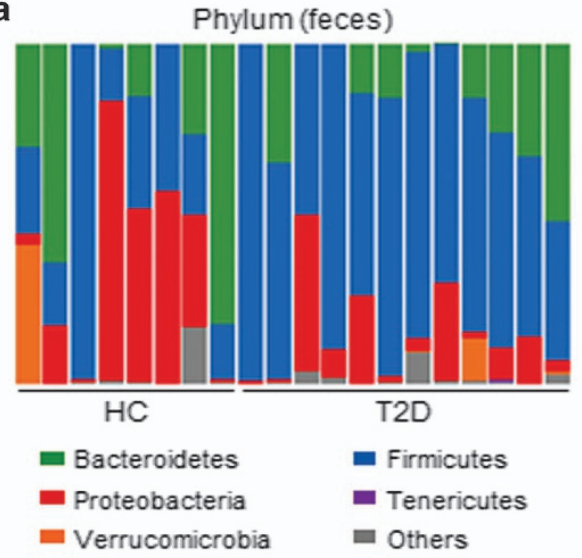

b

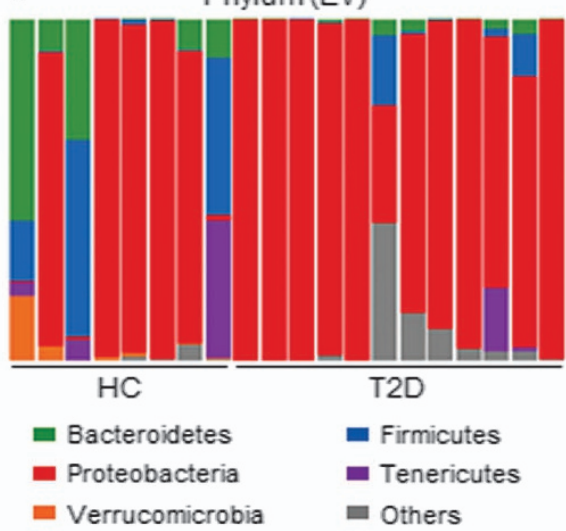

c

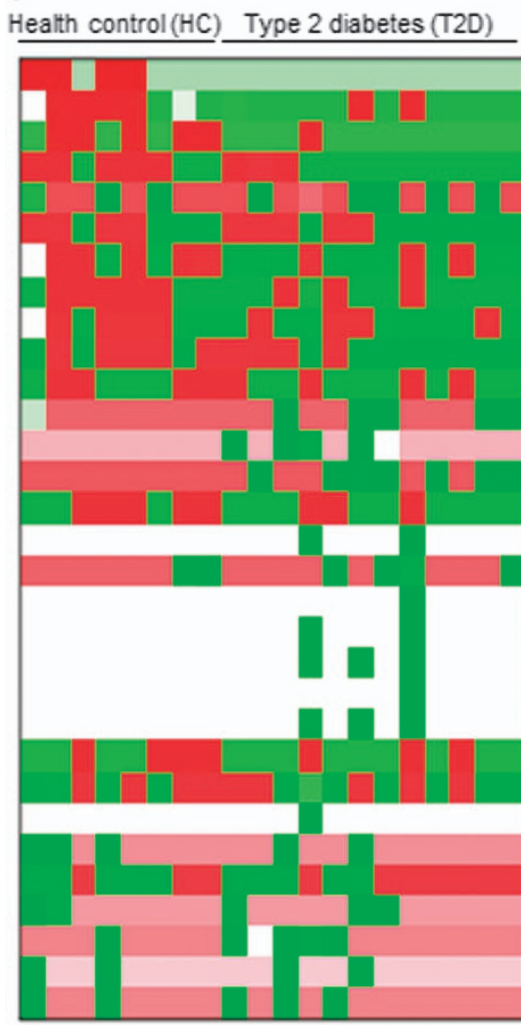

Species sorted

by $\mathrm{HC} / \mathrm{T} 2 \mathrm{D}$ ratio

Akkermansia muciniphila

Staphylococcus epidermidis

Rhizobiummassiliae

Pseudomonas fuscovaginae

Delftia lacustris

Pseudomonas japonica

Pseudomonas extremorientalis

Pseudomonas orientalis

Pelomonas puraquae

Comamonas testosteroni

Pseudomonas proteolytica

Pseudomonas hibiscicola

Pseudomonas libanensis

Pseudomonas panacis

Pseudomonas geniculata

Pseudomonas marginalis

Propionibacterium acnes

Pseudomonas trivialis

Pseudomonas cedrina

Pseudomonas mediterranea

Pseudomonas rhodesiae

Pseudomonas grimontii

Pseudomonas brenneri

Rothia mucilaginosa

Pseudomonas extremaustralis

Brevundimonas vesicularis

Brevundimonas aurantiaca

Phyllobacterium myrsinacearum

Rhodococcus qingshengii

Brevundimonas intermedia

Microbacterium maritypicum

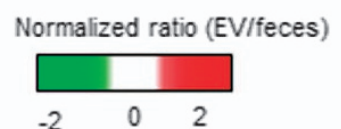

Figure 1 Microbial diversity and relative abundances of EVs in human feces. Metagenomic data showing microbial diversity. (a) Microbial diversity in the feces of type 2 diabetic patients and healthy controls. (b) Microbial diversity in the fecal EVs of type 2 diabetic patients and healthy controls. (c) Heat map of normalized EV contents. Only species that showed significant differences were used to draw this heat map $(P<0.05)$. The order of species was sorted by the content ratio (type 2 diabetes patients over healthy controls), showing that $A$. muciniphila might be a candidate EV-secreting bacterium that suppresses type 2 diabetes.

\section{Isolation and characterization of AmEVs}

To understand the role played by AmEVs, we next isolated AmEVs and compared them with E. coli-derived EVs (EcEVs). Pathogenic strains of E. coli are abundant in individuals suffering from intestinal inflammation and are known to induce intestinal permeability in vivo and in vitro; hence, they were used as a control in our study. ${ }^{33-35}$ Transmission electron microscopic analysis revealed that both AmEVs and EcEVs were spherical in shape and had lipid bilayers (Supplementary Figure S2A). To further characterize the size of the individual
EVs, these vesicles were analyzed using dynamic light scattering. AmEVs and EcEVs had very similar diameters, with a peak size range of 40-60 nm (Supplementary Figure S2B). To assess the protein content of the EVs, simple SDS-PAGE analysis was performed. As expected, the protein band patterns were different, reflecting a distinct composition of proteins in each EV (Supplementary Figure S2C). These results suggest that physical characteristics such as shape and size alone cannot distinguish AmEVs from EcEVs but that the molecular compositions of the EVs are different. 
a

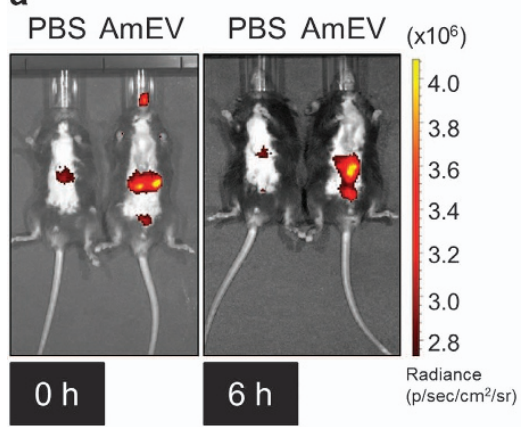

b

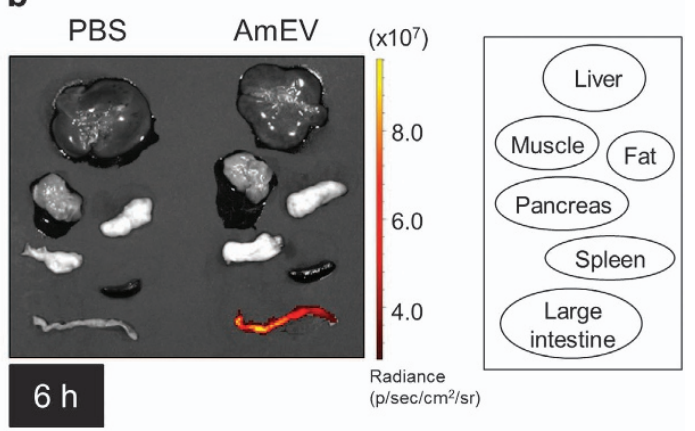

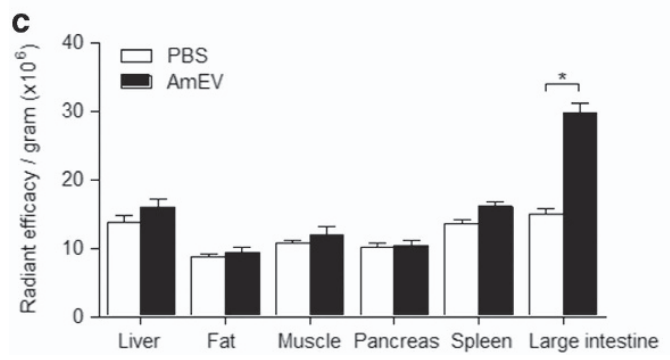

Figure 2 In vivo distribution of Cy7-labeled A. muciniphila-derived EVs in mice. Cy7-labeled AmEVs (50 $\mu$ g of total protein) were orally administered to C57BL/6 mice. (a) In vivo fluorescent whole-body images of AmEVs in mice were acquired with an IVIS spectrum. (b) Ex vivo image of various tissues, including liver, fat, muscle, pancreas, spleen and large intestine, after $6 \mathrm{~h}$ of EV feeding. (c) Radiant efficiency was measured using the Living Image 3.1 software. All data are presented as the mean \pm s.e.m. of 3 experiments, with $n=5-7$ animals per group for each experiment. ${ }^{*} P<0.05$.

\section{Distribution of AmEVs after oral administration}

To understand the mechanism of action of EVs in regulating metabolic functions, we checked the distribution of AmEVs in peripheral tissues. AmEVs conjugated with $\mathrm{Cy} 7$, a near infrared dye, were injected orally into $\mathrm{C} 57 \mathrm{BL} / 6$ mice, and the $\mathrm{EV}$ distribution was examined using near-infrared imaging. After $6 \mathrm{~h}$, in vivo imaging revealed that AmEVs had spread throughout the mouse body (Figure 2a). Subsequently, the organs were harvested, and Cy7 signaling in the liver, fat, muscle and other organs was observed (Figure 2b). Ex vivo images were analyzed by radiant efficiency (Figure 2c). These results suggest that most of the AmEVs can translocate into the large intestine within $6 \mathrm{~h}$ of feeding.

AmEVs improve gut permeability in HFD-fed mice through the regulation of tight junctions

To examine the role of AmEVs in vivo, an HFD-fed diabetic mouse model was employed. C57BL/6 mice were fed either normal chow diet (NCD) or HFD for 10 weeks, followed by oral gavaging of AmEVs every other day for 2 weeks (Supplementary Figure S3A). AmEV feeding significantly reduced HFD-induced body weight gain in these mice (Figure 3a). To verify whether AmEV administration induced any changes in in vivo intestinal permeability, the animals were fasted for $8 \mathrm{~h}$, followed by oral gavaging with $400 \mathrm{mg} \mathrm{kg}^{-1}$ FITC-labeled dextran. The serum FITC concentration was measured by spectrofluorometry. HFD feeding significantly increased gut permeability, as indicated by the increased serum FITC concentration, which was reduced in AmEV-fed animals (Figure 3b). HFD feeding drastically reduced the colon length in mice, and this was also partially ameliorated after AmEV feeding for 2 weeks (Figures $3 \mathrm{c}$ and d). These results show that HFD induces severe damage in the intestine that affects its integrity, which can be treated by AmEV feeding. We further examined colon sections from these animals. Hematoxylin and eosin staining of the colon sections revealed significant damage to the barrier structure in HFD-fed mice accompanied by the recruitment of immune cells into the intestinal epithelial layer. However, in the HFD+AmEV-fed group, damage to the intestinal epithelial layer was significantly lower (Figure 3e, Supplementary Figure S3B). Because the intestinal barrier permeability is modified by altered tight junction protein expression, we examined the expression of occludin, zonal occludens and claudin-5, three major tight junction proteins that are responsible for the maintenance of barrier integrity. ${ }^{36}$ In concurrence with our intestinal permeability data, HFD feeding significantly reduced the expression of these proteins in the intestinal epithelial layer, and the expression was increased in the HFD+AmEV-fed group (Figure 3f, Supplementary Figure S3C). HFD-induced intestinal permeability is thought to be a crucial factor for metabolic endotoxemia and diabetes onset. To verify whether AmEVs had any protective effect on HFD-induced diabetes, we performed a glucose tolerance test. AmEV treatment significantly improved glucose tolerance in mice compared with that in mice fed with an HFD alone, indicating that AmEV treatment was able to improve metabolic function in HFD-induced diabetic mice (Supplementary Figure S3D). Overall, these results reveal that AmEV feeding can protect mice from HFD-induced gut permeability and improve metabolic functions. 
a

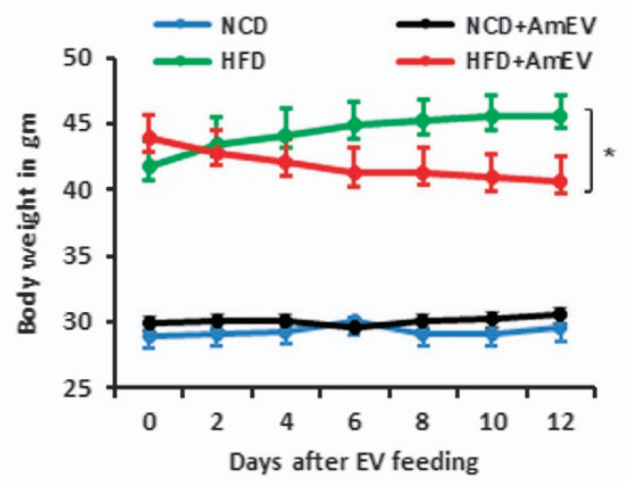

C

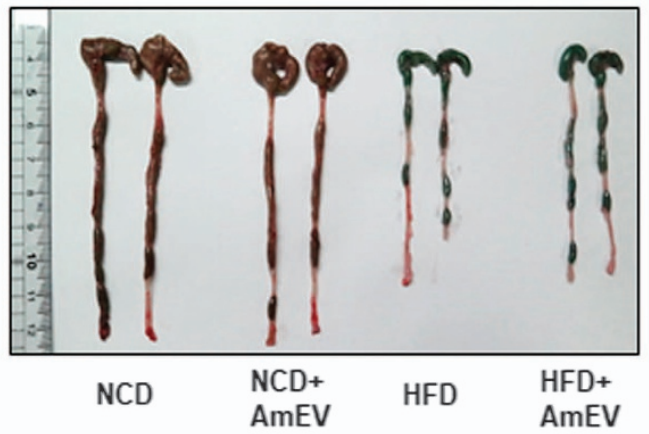

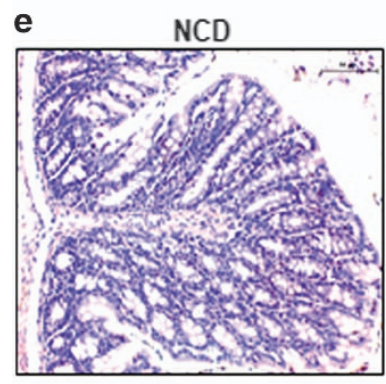

HFD

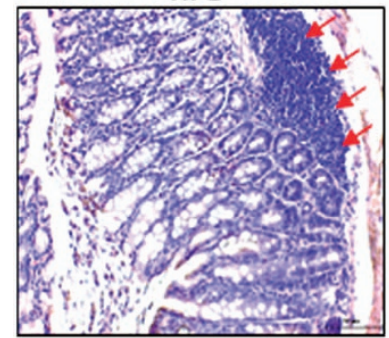

$\mathrm{NCD}+\mathrm{AmEV}$

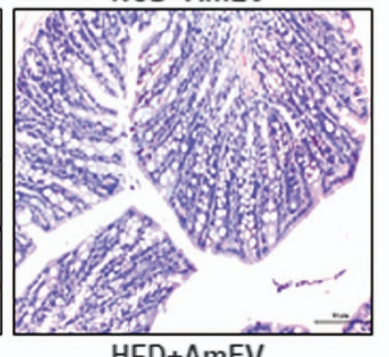

HFD+AmEV

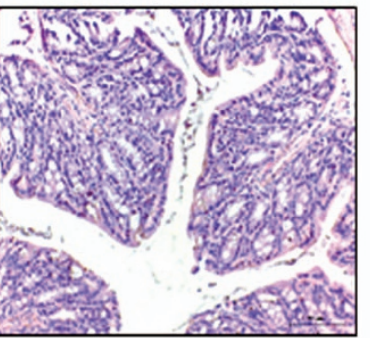

b

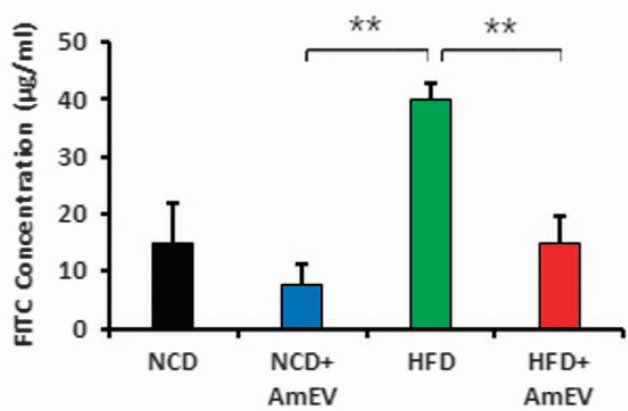

d

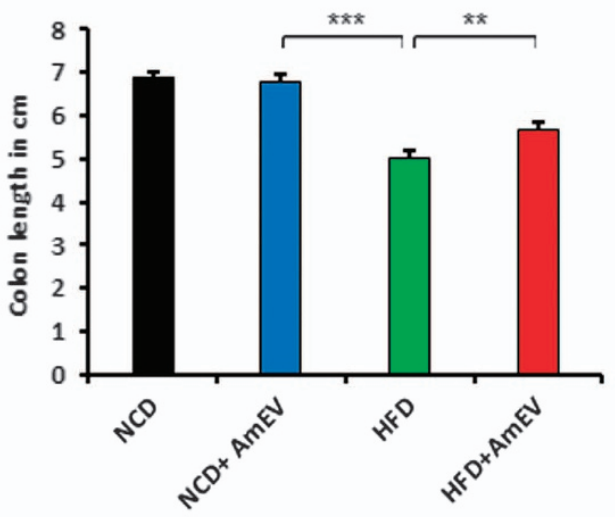

f

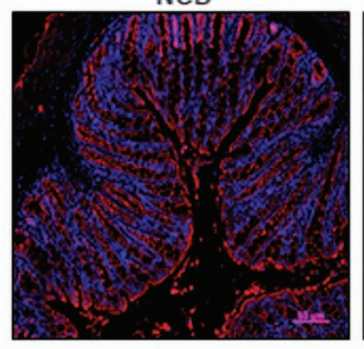

HFD

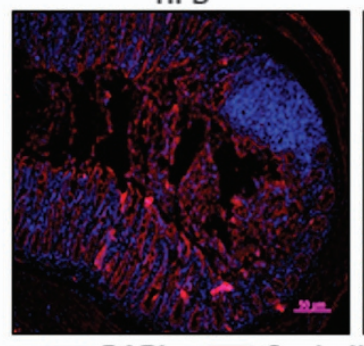

DAPI Occludin

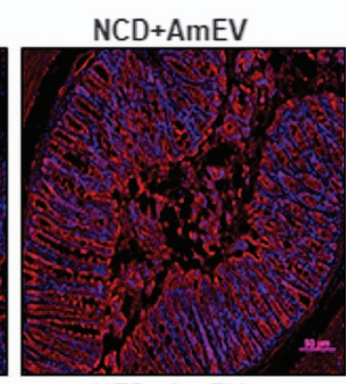

HFD+AmEV

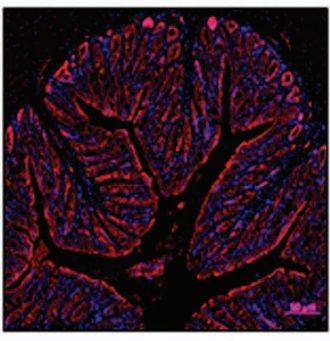

Figure 3 A. muciniphila-derived EVs improve gut permeability in high-fat diet (HFD)-fed mice. Mice were fed a normal chow diet (NCD) or a HFD for 12 weeks, followed by oral administration of AmEVs (10 $\mu$ g per mouse) every other day for 2 weeks. (a) Body weight changes were measured at the indicated time points. The graph shows body weight change from the day that EV feeding started. (b) In vivo intestinal permeability assay in NCD, NCD+AmEV, HFD and HFD+AmEV mice conducted after $4 \mathrm{~h}$ of treatment with FITC-dextran. The serum FITC concentration was measured using blood collected by retro-orbital bleeding, and fluorescence was measured using a spectrofluorometer. Data are shown as the mean \pm s.e.m. (c) Gross imaging of the colons dissected from NCD-, NCD+AmEV-, HFD- and HFD+AmEV-fed mice. (d) The colon length of NCD-, NCD+AmEV-, HFD- and HFD+AmEV-fed mice. (e) Hematoxylin and eosin staining of colon sections from NCD-, NCD+AmEV-, HFD- and HFD+AmEV-fed mice. Red arrows indicate the recruitment of immune cells. Scale bar is $50 \mu \mathrm{m}$. (f) Immunohistochemical images showing occludin expression (occludin-red, nucleus-blue) in NCD-, NCD+AmEV-, HFD- and $\mathrm{HFD}+$ AmEV-fed mice. Scale bar is $50 \mu \mathrm{m}$. All data are presented as the mean \pm s.e.m. of 3 experiments; $n=5-7$ per group; ${ }^{*} P<0.05$, $* * P<0.01$. 
AmEVs improve barrier integrity in Caco-2 cells through AMPK activation

To verify the mechanism of EVs in tight junction regulation, we performed in vitro permeability assays using monolayer cultures of Caco-2 cells. High-calorie and high-fat western-style diets alter the gut microbial composition and increase the level

a

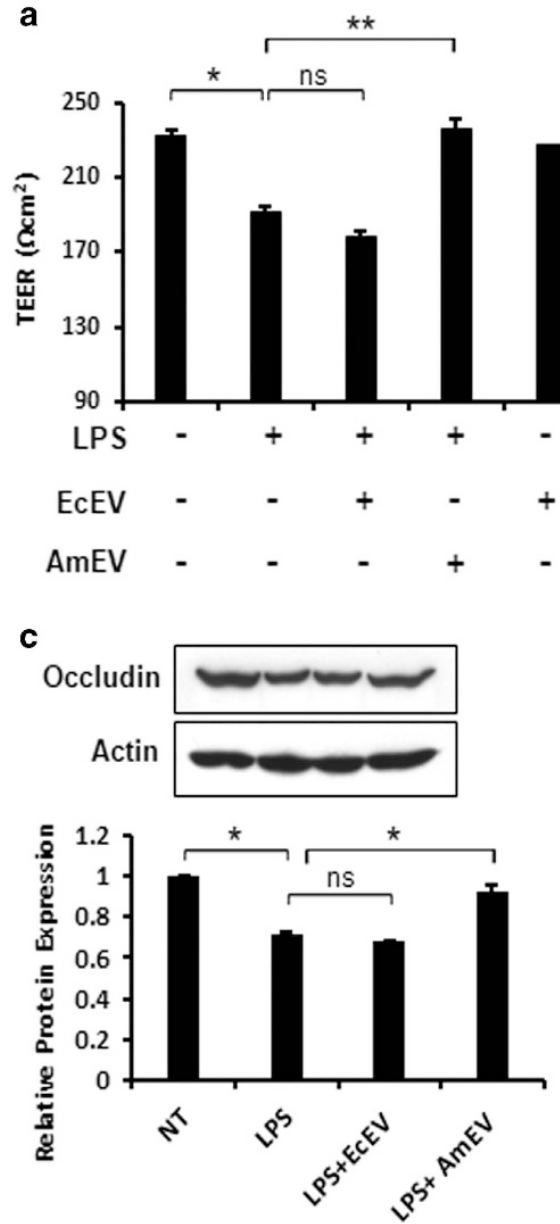

e
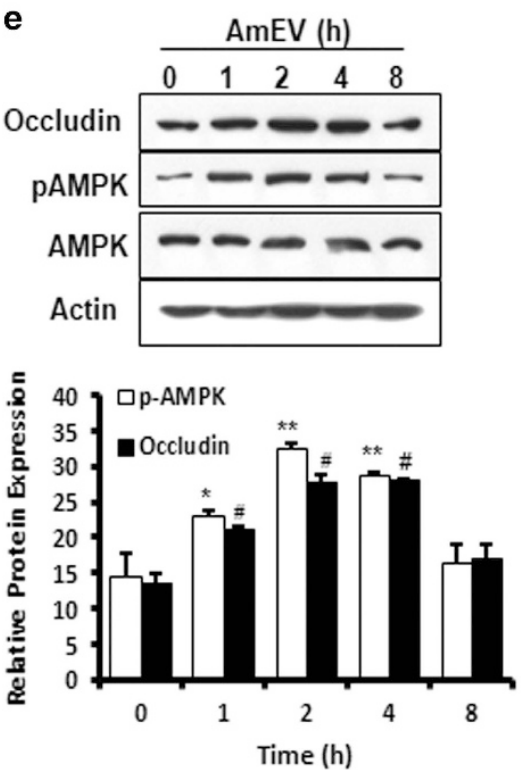

of gut microbiome-derived endotoxin or LPS in circulation. ${ }^{14,37,38}$ Increased metabolic endotoxemia is thought to be due to major changes in the gut microbiome, and the gut-derived LPS could be responsible for the increase in intestinal permeability. ${ }^{39}$ To mimic this, Caco-2 transwell monolayer cultures were treated with LPS alone, EcEVs or

b

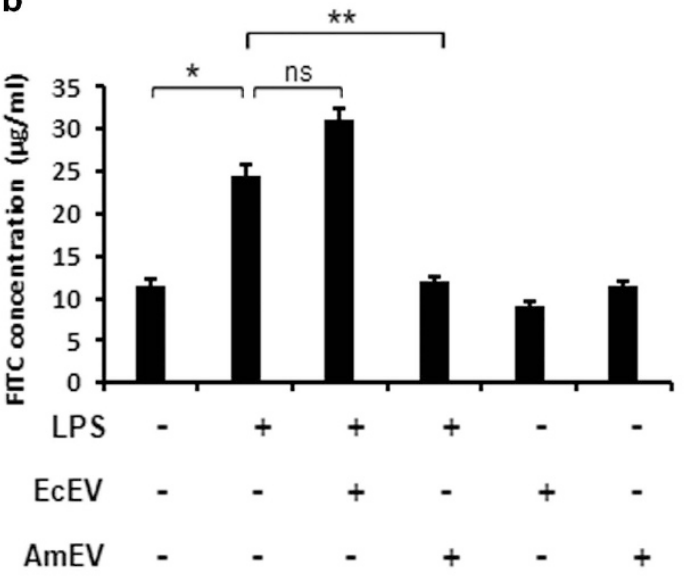

d
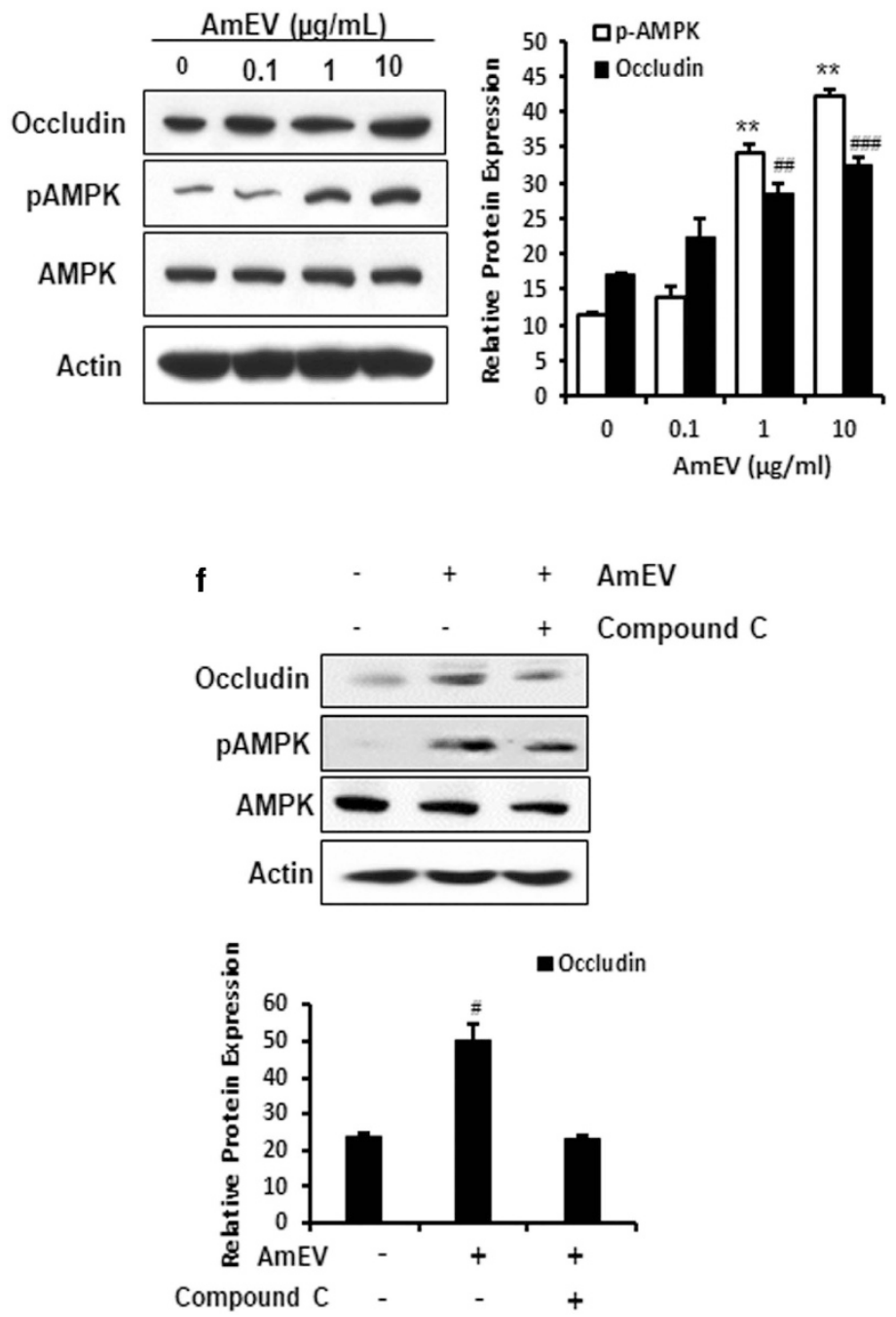
AmEVs. The decrease in the trans-epithelial electrical resistance induced by LPS was rescued by the administration of AmEVs (Figure 4a). The FITC-dextran permeability assay in Caco-2 cells also confirmed that AmEV treatment could improve intestinal barrier integrity under LPS treatment conditions (Figure 4b). We observed that only AmEVs and not EcEVs decreased LPS-induced tight junction permeability (Figures 4a and $\mathrm{b}$ ).

Tight junction proteins are major factors that contribute to intestinal barrier integrity. Analysis of tight junction protein expression by immunoblotting revealed that the LPS-induced decrease in occludin expression was ameliorated by AmEV treatment but remained the same after EcEV treatment (Figure 4c). To understand the mechanism of the regulation of tight junction permeability, AmEVs and EcEVs were administered to Caco-2 cells in a dose- and time-dependent manner. We found that AmEVs induced phosphorylation of AMPK, a kinase responsible for tight junction re-assembly and stability, ${ }^{40-42}$ in a dose-dependent manner but that EcEVs did not. AMPK phosphorylation increased steadily following AmEV treatment in a dose-dependent manner compared with EcEVs (Figure 4d,Supplementary Figure S4A). AMPK activation occurred within $1 \mathrm{~h}$ of AmEV administration; however, p-AMPK expression was not changed after EcEV administration (Figure 4e,Supplementary Figure S4B). AmEV treatment also improved intestinal barrier integrity in a dose-dependent manner (Supplementary Figure S4C). Furthermore, to confirm the role of AMPK in AmEV-induced tight junction regulation, Caco-2 cells were co-treated with AmEV and AMPK inhibitor compound C. AmEV treatment could not induce the expression of occludin in the presence of AMPK inhibitor compound C (Figure 4f). Similarly, AmEVs' ability to improve LPSinduced loss of barrier integrity in vitro was abolished in the presence of compound C (Supplementary Figures S4D and E). Collectively, these data suggest that AmEVs are potent AMPK activators that decrease tight junction permeability in Caco-2 cells. Our results suggest that AmEVs activate the AMPK pathway, which is responsible for the regulation of tight junctions.

\section{DISCUSSION}

In the present study, we demonstrated the regulatory effect of AmEVs on intestinal permeability via the regulation of the tight junction protein occludin through AMPK activation. Moreover, AmEV-treated diabetic mice exhibited improved metabolic profiles, including body weight and glucose tolerance. We also observed that AmEVs were enriched in the feces of healthy controls compared with those of T2D patients, indicating a potential role for AmEVs as a therapeutic intervention for obesity and diabetes.

Maintenance of intestinal barrier integrity is crucial for the proper physiology and homeostasis in the human body. ${ }^{43-45} \mathrm{~A}$ compromised intestinal barrier integrity can be detrimental ${ }^{46}$ and lead to the onset of several gastro-intestinal, ${ }^{47-49}$ metabolic $^{13,50,51}$ and neurological disorders. ${ }^{52,53}$ Previous studies have reported a close association between obesity and the loss of barrier integrity. Increased intestinal barrier permeability induces metabolic endotoxemia, which is thought to be a major factor contributing to metabolic disorders in obesity. ${ }^{54} \mathrm{~A}$ leaky gut allows the passage of gut microbiota-derived LPS into the blood stream, leading to metabolic endotoxemia, a trigger for onset of obesity and diabetes. Though it is not clear whether the loss of barrier integrity is an initial event in the pathogenesis of obesity and T2D, a myriad of experimental and clinical studies reinforces the importance of the maintenance of gut barrier integrity. Consequently, regulating intestinal barrier integrity is gaining attention as an intriguing therapeutic strategy ${ }^{55,56}$ for metabolic disorders.

The gut microbiota is now widely investigated for its role in regulating intestinal barrier integrity, host energy balance and homeostasis by modulating host signaling pathways. ${ }^{57,58} \mathrm{~A}$. muciniphila, a beneficial gut bacterium, has been previously identified to be inversely correlated with body weight in type 2

Figure $4 \mathrm{~A}$. muciniphila-derived EVs reduce LPS-induced intestinal permeability through AMPK phosphorylation. (a) Trans-epithelial

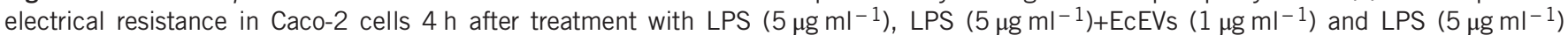

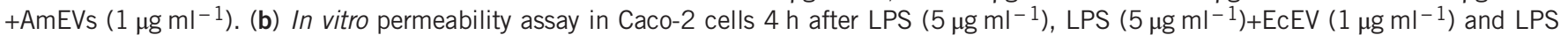

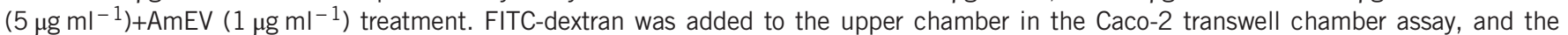
transport of FITC-labeled dextran across the Caco-2 monolayer culture was measured by fluorescence spectrofluorometry. (c) Expression of tight junction proteins in Caco-2 cells after LPS, LPS+EcEV and LPS+AmEV treatments. A graph showing the relative occludin expression (normalized to actin) is also shown $\left({ }^{*} P<0.05\right)$. (d) AmEV treatment increased occludin expression and phosphorylation of AMPK 22 thr172 in a dose-dependent manner. Caco-2 cells were treated with $0.1,1$ and $10 \mu \mathrm{g} \mathrm{ml}^{-1}$ AmEVs, and the expression of occludin, $\mathrm{p}$-AMPK, total AMPK and actin was analyzed after $4 \mathrm{~h}$ by immunoblotting. A graph showing the relative protein expression normalized to actin is

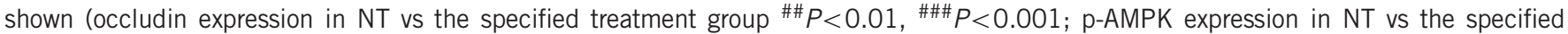
treatment group $* * P<0.01$ ). (e) Time course of AmEV-induced occludin and p-AMPK expression in Caco-2 cells. Caco-2 cells were treated with $1 \mu \mathrm{g} \mathrm{ml} \mathrm{m}^{-1}$ AmEVs, and the expression of occludin, p-AMPK, total AMPK and actin was analyzed after $1,2,4$ and $8 \mathrm{~h}$ by immunoblotting. The graph shows the relative protein expression after normalization to actin (occludin expression in NT vs the specified time point ${ }^{\#} P<0.05$; p-AMPK expression in NT vs the specified time point ${ }^{*} P<0.05$, ${ }^{*} P<0.01$ ). (f) Western blot showing the effect of the AMPK inhibitor on AmEV-treated Caco-2 cells. Caco-2 cells were treated with the AMPK-specific inhibitor compound $\mathrm{C}$ for 30 min, after which $1 \mu \mathrm{g} \mathrm{ml}^{-1}$ AmEVs was applied. Protein expression of occludin and p-AMPK was observed after $4 \mathrm{~h}$. The graph shows the relative occludin expression after normalization to actin (occludin expression in NT vs the specified time point ${ }^{\#} P<0.05$ ). All data are presented as the mean \pm s.e.m. of three experiments performed in triplicate. NS, not significant. 


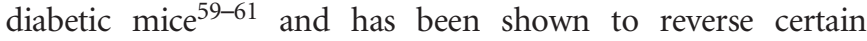
metabolic parameters, such as insulin resistance. ${ }^{9}$ An outer membrane protein of A. muciniphila, Amuc_1100, which interacts with host Toll-like receptor 2, was recently found to have an important role in promoting gut barrier integrity through the upregulation of tight junction proteins and is thought to be involved in the beneficial properties shown by this bacterium in patients with obesity and diabetes. ${ }^{62,63}$ However, the complexities involved in culturing these bacteria limit their clinical application.

Here we suggest that EVs, complex biological molecules that mediate interactions between the gut microbiota and the host, can be potent components for the regulation of intestinal barrier integrity by the gut microbiome. ${ }^{64-66} \mathrm{EVs}$ are spherical lipid bilayer structures ${ }^{67}$ released from bacteria and can enter the host systemic circulation and induce immunological and metabolic responses by delivering their components into host cells and possibly interacting with specific intracellular compartments. ${ }^{68,69}$ We found that EVs derived from $A$. muciniphila were responsible for improving intestinal barrier integrity. We also showed that AmEVs improved body weight and glucose tolerance in diabetic mice (Figure 3). AmEV treatment reduced HFD-induced barrier permeability. It is likely that the resulting improvement in metabolic endotoxemia could have resulted in the improved metabolic phenotypes, such as glucose tolerance and body weight. Previous studies have shown that the feeding of A. muciniphila to HFDfed mice can improve metabolic endotoxemia.9,70 However, until now, little has been known about the direct effects of EVs on cellular physiology and intestinal barrier regulation. Here we show that EVs derived from A. muciniphila could be responsible for the beneficial effects shown by this bacterium. Therefore, this study provides unique and significant insights into the influence of EVs on the host and deepens our current understanding of the role the gut microbiome has in regulating gut barrier integrity. It should also be noted that AmEVs may improve the metabolic phenotypes in HFD-fed mice through other mechanisms. Further in-depth studies are imperative to decipher the other roles of AmEVs in improving HFD dietinduced phenotypes.

Furthermore, we demonstrate that AmEVs can activate AMPK, which is involved in tight junction regulation. The role of AMPK activation in fortifying epithelial tight junctions has been widely studied. ${ }^{42}$ AMPK is essential for the assembly of tight junction proteins at cell-cell junctions, and initiation of tight junction assembly is compromised in cells lacking active AMPK. ${ }^{41,42}$ EVs derived from $A$. muciniphila promote tight junction expression by activating AMPK. This was proven by the abolishment of the effects of AmEVs after AMPK inhibitor treatment (Figure 4).

However, it is yet to be determined what component of AmEVs activates AMPK. Here we simply visualized the protein banding patterns of AmEVs and EcEVs using SDS-PAGE. Our analysis revealed different protein contents in the two different EVs, and this difference may contribute to the varying effects of each EV, as proteins are typically the functional component.
However, proteins are not the only molecules that can influence the physiological condition of the cells. Therefore, finding the effective component in the EVs is a challenging task, as these vesicles are composed of several molecules, including proteins, lipids, nucleic acids and other factors. ${ }^{71,72}$ Proteomic or lipidomic experiments would help to identify the specific molecule or molecules in AmEVs that contribute to the regulation of intestinal barrier integrity.

Here we focused on the role of EVs in promoting intestinal barrier integrity. Convincing evidence supporting a role for EVs as arbitrators of microbiome-host signaling is only starting to emerge. In this study, for the first time, we identified a potential role for EVs from A. muciniphila in improving gut barrier integrity under pathophysiological conditions induced by high-calorie eating habits. We observed that AmEVs from gavage penetrate the large intestine and spread to peripheral tissues. However, our results do not verify whether EVs remained intact after traveling throughout the body. Moreover, there are no data on the ratio of EVs in the gut to EVs in peripheral tissues. It is important to measure these circulating vesicles in order to determine the efficiency of EV influence on host cells. It would also be interesting to investigate the role of EVs in peripheral tissues such as muscle, adipose tissue and liver and how EV-host interactions at the tissue level contribute to physiology and homeostasis. Thus further investigations to identify the pathways of EVs will provide a more thorough understanding of the mechanisms underlying the complex role of EVs in physiological and pathophysiological conditions.

\section{CONFLICT OF INTEREST}

The authors declare no conflict of interest.

\section{ACKNOWLEDGEMENTS}

This work was supported by a National Research Foundation of Korea (NRF) grant funded by the Korean government (MEST) (No.

NRF-2016K1A1A2912722), the Ministry of Health and Welfare (No. HI14C0306) and the Samsung Research Funding Center of Samsung Electronics (SRFC-MA1502-14).

Author contributions: CC and YC designed the experiments, analyzed the data and wrote the manuscript; D-KK analyzed the clinical metagenomic data and wrote/revised the manuscript; HTP, YK and JJ performed the experiments; M-SK, Y-KJ, YSG, YK, JG and SHR designed this project and provided directions for the manuscript. All authors reviewed and approved this manuscript.

\section{PUBLISHER'S NOTE}

Springer Nature remains neutral with regard to jurisdictional claims in published maps and institutional affiliations.

1 Cani PD, Osto M, Geurts L, Everard A. Involvement of gut microbiota in the development of low-grade inflammation and type 2 diabetes associated with obesity. Gut Microbes 2012; 3: 279-288.

2 Cani PD, Amar J, Iglesias MA, Poggi M, Knauf C, Bastelica D et al. Metabolic endotoxemia initiates obesity and insulin resistance. Diabetes 2007; 56: 1761-1772. 
3 Osborn 0, Olefsky JM. The cellular and signaling networks linking the immune system and metabolism in disease. Nat Med 2012; 18: 363-374.

4 Collado MC, Isolauri E, Laitinen K, Salminen S. Distinct composition of gut microbiota during pregnancy in overweight and normal-weight women. Am J Clin Nutr 2008; 88: 894-899.

5 Karlsson CL, Onnerfalt J, Xu J, Molin G, Ahrne S, Thorngren-Jerneck K. The microbiota of the gut in preschool children with normal and excessive body weight. Obesity (Silver Spring) 2012; 20: 2257-2261.

6 Belzer C, de Vos WM. Microbes inside-from diversity to function: the case of Akkermansia. ISME J 2012; 6: 1449-1458.

7 Derrien M, Vaughan EE, Plugge CM, de Vos WM. Akkermansia muciniphila gen. nov., sp. nov., a human intestinal mucin-degrading bacterium. Int $J$ Syst Evol Microbiol 2004; 54: 1469-1476.

8 Collado MC, Derrien M, Isolauri E, de Vos WM, Salminen S. Intestinal integrity and Akkermansia muciniphila, a mucin-degrading member of the intestinal microbiota present in infants, adults, and the elderly. App/ Environ Microbiol 2007; 73: 7767-7770.

9 Everard A, Belzer C, Geurts L, Ouwerkerk JP, Druart C, Bindels LB et al. Cross-talk between Akkermansia muciniphila and intestinal epithelium controls diet-induced obesity. Proc Natl Acad Sci USA 2013; 110: 9066-9071.

10 Derrien M, Van Baarlen P, Hooiveld G, Norin E, Muller M, de Vos WM. Modulation of mucosal immune response, tolerance, and proliferation in mice colonized by the mucin-degrader Akkermansia muciniphila. Front Microbiol 2011; 2: 166.

11 Konig J, Wells J, Cani PD, Garcia-Rodenas CL, MacDonald T, Mercenier A et al. Human Intestinal Barrier Function in Health and Disease. Clin Transl Gastroenterol 2016; 7: e196.

12 Moreira AP, Texeira TF, Ferreira AB, Peluzio Mdo C, Alfenas Rde C. Influence of a high-fat diet on gut microbiota, intestinal permeability and metabolic endotoxaemia. Br J Nutr 2012; 108: 801-809.

13 Cani PD, Possemiers S, Van de Wiele T, Guiot Y, Everard A, Rottier 0 et al. Changes in gut microbiota control inflammation in obese mice through a mechanism involving GLP-2-driven improvement of gut permeability. Gut 2009; 58: 1091-1103.

14 Cani PD, Bibiloni R, Knauf C, Waget A, Neyrinck AM, Delzenne NM et al. Changes in gut microbiota control metabolic endotoxemia-induced inflammation in high-fat diet-induced obesity and diabetes in mice. Diabetes 2008; 57: 1470-1481.

15 Ellis TN, Kuehn MJ. Virulence and immunomodulatory roles of bacterial outer membrane vesicles. Microbiol Mol Biol Rev 2010; 74: 81-94.

16 Lee EY, Choi DY, Kim DK, Kim JW, Park JO, Kim S et al. Gram-positive bacteria produce membrane vesicles: proteomics-based characterization of Staphylococcus aureus-derived membrane vesicles. Proteomics 2009; 9: 5425-5436.

17 Lee EY, Bang JY, Park GW, Choi DS, Kang JS, Kim HJ et al. Global proteomic profiling of native outer membrane vesicles derived from Escherichia coli. Proteomics 2007; 7: 3143-3153.

18 Horstman AL, Kuehn MJ. Bacterial surface association of heat-labile enterotoxin through lipopolysaccharide after secretion via the general secretory pathway. J Biol Chem 2002; 277: 32538-32545.

19 Hong SW, Kim MR, Lee EY, Kim JH, Kim YS, Jeon SG et al. Extracellular vesicles derived from Staphylococcus aureus induce atopic dermatitis-like skin inflammation. Allergy 2011; 66: 351-359.

20 Kim YS, Choi EJ, Lee WH, Choi SJ, Roh TY, Park J et al. Extracellular vesicles, especially derived from Gram-negative bacteria, in indoor dust induce neutrophilic pulmonary inflammation associated with both Th1 and Th17 cell responses. Clin Exp Allergy 2013; 43: 443-454.

21 Kuehn MJ, Kesty NC. Bacterial outer membrane vesicles and the hostpathogen interaction. Genes Dev 2005; 19: 2645-2655.

22 Choi Y, Kwon Y, Kim DK, Jeon J, Jang SC, Wang T et al. Gut microbederived extracellular vesicles induce insulin resistance, thereby impairing glucose metabolism in skeletal muscle. Sci Rep 2015; 5: 15878.

23 Kang CS, Ban M, Choi EJ, Moon HG, Jeon JS, Kim DK et al. Extracellular vesicles derived from gut microbiota, especially Akkermansia muciniphila, protect the progression of dextran sulfate sodium-induced colitis. PLOS ONE 2013; 8: e76520.

24 Ma TY, Hoa NT, Tran DD, Bui V, Pedram A, Mills S et al. Cytochalasin B modulation of Caco-2 tight junction barrier: role of myosin light chain kinase. Am J Physiol Gastrointest Liver Physiol 2000; 279: G875-G885

25 Natoli M, Leoni BD, D'Agnano I, Zucco F, Felsani A. Good Caco-2 cell culture practices. Toxicol In Vitro 2012; 26: 1243-1246.
26 Chelakkot C, Ghim J, Rajasekaran N, Choi JS, Kim JH, Jang MH et al. Intestinal epithelial cell-specific deletion of PLD2 alleviates DSS-induced colitis by regulating occludin. Sci Rep 2017; 7: 1573.

27 Biganzoli E, Cavenaghi LA, Rossi R, Brunati MC, Nolli ML. Use of a Caco-2 cell culture model for the characterization of intestinal absorption of antibiotics. Farmaco 1999; 54: 594-599.

28 Yamashita S, Furubayashi T, Kataoka M, Sakane T, Sezaki H, Tokuda H. Optimized conditions for prediction of intestinal drug permeability using Caco-2 cells. Eur J Pharm Sci 2000; 10: 195-204.

29 Corr SC, Palsson-McDermott EM, Grishina I, Barry SP, Aviello G, Bernard $\mathrm{NJ}$ et al. MyD88 adaptor-like (Mal) functions in the epithelial barrier and contributes to intestinal integrity via protein kinase C. Mucosal Immunol 2014; 7: 57-67.

30 Wang Q, Pantzar N, Jeppsson B, Westrom BR, Karlsson BW. Increased intestinal marker absorption due to regional permeability changes and decreased intestinal transit during sepsis in the rat. Scand J Gastroenterol 1994; 29: 1001-1008.

31 Brandl K, Rutschmann S, Li X, Du X, Xiao N, Schnabl B et al. Enhanced sensitivity to DSS colitis caused by a hypomorphic Mbtps 1 mutation disrupting the ATF6-driven unfolded protein response. Proc Natl Acad Sci USA 2009; 106: 3300-3305.

32 Hwang D, Rust AG, Ramsey S, Smith JJ, Leslie DM, Weston AD et al. A data integration methodology for systems biology. Proc Natl Acad Sci USA 2005; 102: 17296-17301.

33 Kim YS, Lee WH, Choi EJ, Choi JP, Heo YJ, Gho YS et al. Extracellular vesicles derived from Gram-negative bacteria, such as Escherichia coli, induce emphysema mainly via IL-17A-mediated neutrophilic inflammation. J Immunol 2015; 194: 3361-3368.

34 Park KS, Choi KH, Kim YS, Hong BS, Kim OY, Kim JH et al. Outer membrane vesicles derived from Escherichia coli induce systemic inflammatory response syndrome. PLOS ONE 2010; 5: e11334.

35 Mangell P, Nejdfors $\mathrm{P}$, Wang M, Ahrne S, Westrom B, Thorlacius $\mathrm{H}$ et al. Lactobacillus plantarum 299v inhibits Escherichia coli-induced intestinal permeability. Dig Dis Sci 2002; 47: 511-516.

36 Feldman GJ, Mullin JM, Ryan MP. Occludin: structure, function and regulation. Adv Drug Deliv Rev 2005; 57: 883-917.

37 Wellen KE, Hotamisligil GS. Inflammation, stress, and diabetes. J Clin Invest 2005; 115: 1111-1119.

38 Mehta NN, McGillicuddy FC, Anderson PD, Hinkle CC, Shah R, Pruscino L et al. Experimental endotoxemia induces adipose inflammation and insulin resistance in humans. Diabetes 2010; 59: 172-181.

39 Hadad SM, Appleyard V, Thompson AM. Therapeutic metformin/AMPK activation promotes the angiogenic phenotype in the ERalpha negative MDA-MB-435 breast cancer model. Breast Cancer Res Treat 2009; 114: 391.

40 Zhang L, Jouret F, Rinehart J, Sfakianos J, Mellman I, Lifton RP et al. AMPactivated protein kinase (AMPK) activation and glycogen synthase kinase3beta (GSK-3beta) inhibition induce Ca2+-independent deposition of tight junction components at the plasma membrane. J Biol Chem 2011; 286: 16879-16890.

41 Zheng B, Cantley LC. Regulation of epithelial tight junction assembly and disassembly by AMP-activated protein kinase. Proc Natl Acad Sci USA 2007; 104: 819-822.

42 Zhang L, Li J, Young LH, Caplan MJ. AMP-activated protein kinase regulates the assembly of epithelial tight junctions. Proc Natl Acad Sci USA 2006; 103: 17272-17277.

43 Wells JM, Brummer RJ, Derrien M, MacDonald TT, Troost F, Cani PD et al. Homeostasis of the gut barrier and potential biomarkers. Am J Physiol Gastrointest Liver Physiol 2017; 312: G171-G193.

44 Turner JR. Intestinal mucosal barrier function in health and disease. Nat Rev Immunol 2009; 9: 799-809.

45 Peterson LW, Artis D. Intestinal epithelial cells: regulators of barrier function and immune homeostasis. Nat Rev Immunol 2014; 14: 141-153.

46 Shen L, Su L, Turner JR. Mechanisms and functional implications of intestinal barrier defects. Dig Dis 2009; 27: 443-449.

47 Fasano A, Shea-Donohue T. Mechanisms of disease: the role of intestinal barrier function in the pathogenesis of gastrointestinal autoimmune diseases. Nat Clin Pract Gastroenterol Hepatol 2005; 2: 416-422.

48 Piche T, Barbara G, Aubert P, Bruley des Varannes S, Dainese R, Nano JL et al. Impaired intestinal barrier integrity in the colon of patients with irritable bowel syndrome: involvement of soluble mediators. Gut 2009; 58 : 196-201. 
49 Maloy KJ, Powrie F. Intestinal homeostasis and its breakdown in inflammatory bowel disease. Nature 2011; 474: 298-306.

50 Karl JP, Margolis LM, Madslien EH, Murphy NE, Castellani JW, Gundersen $\mathrm{Y}$ et al. Changes in intestinal microbiota composition and metabolism coincide with increased intestinal permeability in young adults under prolonged physiological stress. Am J Physiol Gastrointest Liver Physiol 2017; 312: G559-G571.

51 Miele L, Valenza V, La Torre G, Montalto M, Cammarota G, Ricci R et al. Increased intestinal permeability and tight junction alterations in nonalcoholic fatty liver disease. Hepatology 2009; 49: 1877-1887.

52 de Magistris L, Familiari V, Pascotto A, Sapone A, Frolli A, lardino P et al. Alterations of the intestinal barrier in patients with autism spectrum disorders and in their first-degree relatives. J Pediatr Gastroenterol Nutr 2010; 51: 418-424.

53 White JF. Intestinal pathophysiology in autism. Exp Biol Med (Maywood) 2003; 228: 639-649.

54 Damms-Machado A, Louis S, Schnitzer A, Volynets V, Rings A, Basrai M et al. Gut permeability is related to body weight, fatty liver disease, and insulin resistance in obese individuals undergoing weight reduction. Am J Clin Nutr 2017; 105: 127-135.

55 Odenwald MA, Turner JR. Intestinal permeability defects: is it time to treat? Clin Gastroenterol Hepatol 2013; 11: 1075-1083.

56 Bischoff SC, Barbara G, Buurman W, Ockhuizen T, Schulzke JD, Serino M et al. Intestinal permeability-a new target for disease prevention and therapy. BMC Gastroenterol 2014; 14: 189.

57 Hildebrandt MA, Hoffmann C, Sherrill-Mix SA, Keilbaugh SA, Hamady M, Chen YY et al. High-fat diet determines the composition of the murine gut microbiome independently of obesity. Gastroenterology 2009; 137 : 1716-1724 e1711-e1712.

58 Backhed F, Manchester JK, Semenkovich CF, Gordon JI. Mechanisms underlying the resistance to diet-induced obesity in germ-free mice. Proc Natl Acad Sci USA 2007; 104: 979-984.

59 Qin J, Li Y, Cai Z, Li S, Zhu J, Zhang F et al. A metagenome-wide association study of gut microbiota in type 2 diabetes. Nature 2012; 490: 55-60.

60 Santacruz A, Collado MC, Garcia-Valdes L, Segura MT, Martin-Lagos JA, Anjos $\mathrm{T}$ et al. Gut microbiota composition is associated with body weight, weight gain and biochemical parameters in pregnant women. Br J Nutr 2010; 104: 83-92.

61 Derrien M, Collado MC, Ben-Amor K, Salminen S, de Vos WM. The Mucin degrader Akkermansia muciniphila is an abundant resident of the human intestinal tract. App/ Environ Microbiol 2008; 74: 1646-1648.

62 Plovier H, Everard A, Druart C, Depommier C, Van Hul M, Geurts L et al. A purified membrane protein from Akkermansia muciniphila or the pasteurized bacterium improves metabolism in obese and diabetic mice. Nat Med 2017; 23: 107-113.
63 Anhe FF, Marette A. A microbial protein that alleviates metabolic syndrome. Nat Med 2017; 23: 11-12.

64 Galan JE, Collmer A. Type III secretion machines: bacterial devices for protein delivery into host cells. Science 1999; 284: 1322-1328.

65 Thery C. Exosomes: secreted vesicles and intercellular communications. F1000 Biol Rep 2011; 3: 15.

66 Ratajczak J, Wysoczynski M, Hayek F, Janowska-Wieczorek A, Ratajczak MZ. Membrane-derived microvesicles: important and underappreciated mediators of cell-to-cell communication. Leukemia 2006; 20 : 1487-1495.

67 Gyorgy B, Szabo TG, Pasztoi M, Pal Z, Misjak P, Aradi B et al. Membrane vesicles, current state-of-the-art: emerging role of extracellular vesicles. Cell Mol Life Sci 2011; 68: 2667-2688.

68 Unal CM, Schaar V, Riesbeck K. Bacterial outer membrane vesicles in disease and preventive medicine. Semin Immunopathol 2011; 33: 395-408.

69 Shen Y, Giardino Torchia ML, Lawson GW, Karp CL, Ashwell JD, Mazmanian SK. Outer membrane vesicles of a human commensal mediate immune regulation and disease protection. Cell Host Microbe 2012; 12 : 509-520.

70 Dao MC, Everard A, Aron-Wisnewsky J, Sokolovska N, Prifti E, Verger EO et al. Akkermansia muciniphila and improved metabolic health during a dietary intervention in obesity: relationship with gut microbiome richness and ecology. Gut 2016; 65: 426-436.

71 Renelli M, Matias V, Lo RY, Beveridge TJ. DNA-containing membrane vesicles of Pseudomonas aeruginosa PAO1 and their genetic transformation potential. Microbiology 2004; 150: 2161-2169.

72 Perez-Cruz C, Delgado L, Lopez-Iglesias C, Mercade E. Outer-inner membrane vesicles naturally secreted by Gram-negative pathogenic bacteria. PLOS ONE 2015; 10: e0116896.

(c) (1) $(\Theta)$ This work is licensed under a Creative Commons Attribution-NonCommercial-NoDerivs 4.0 International License. The images or other third party material in this article are included in the article's Creative Commons license, unless indicated otherwise in the credit line; if the material is not included under the Creative Commons license, users will need to obtain permission from the license holder to reproduce the material. To view a copy of this license, visit http://creativecommons.org/licenses/by-nc-nd/4.0/

(C) The Author(s) 2018

Supplementary Information accompanies the paper on Experimental \& Molecular Medicine website (http://www.nature.com/emm) 